\title{
The sympathetic transmitter norepinephrine inhibits VSMC proliferation induced by TGF $\beta$ by suppressing the expression of the TGF $\beta$ receptor ALK5 in aorta remodeling
}

\author{
ZHIPENG HU, BOWEN LI, ZHIWEI WANG, XIAOPING HU, MIN ZHANG, \\ RUOSHI CHEN, QI WU and FANGYUAN JIA \\ Department of Cardiovascular Surgery, Renmin Hospital of Wuhan University, Wuhan, Hubei 430060, P.R. China
}

Received August 6, 2019; Accepted March 18, 2020

DOI: $10.3892 / \mathrm{mmr} .2020 .11088$

\begin{abstract}
The sympathetic system is involved in the arterial diseases, but its mechanism remains poorly understood. The present study aimed to explore the impact of the sympathetic neurotransmitter norepinephrine (NE) on transforming growth factor (TGF) $\beta$ signaling and the role of NE in aortic remodeling. Guanethidine was used to induce a regional chemical sympathetic denervation (CSD) in angiotensin II (AngII) and $\beta$-aminopropionitrile (BAPN)-induced aortic aneurysm models. The diameter of the aorta was measured, and elastic fiber staining was performed. TGF $\beta$ type I receptor kinase (ALK5) expression in rat aortic NE-treated vascular smooth muscle cells (VSMCs) was detected by reverse transcription-quantitative PCR and western blotting. The effects of NE and ALK5 overexpression on migration, proliferation, apoptosis and TGF $\beta$ signaling were also evaluated. Furthermore, adrenergic receptor blockers were used to determine which receptor was involved in the modulation on TGF $\beta$ signaling by NE. The results of the present study demonstrated that CSD protected rats from AngII+BAPN-induced aortic remodeling and aneurysm formation. Compared with the control group, NE inhibited VSMC proliferation and migration, but promoted apoptosis by suppressing ALK5 expression, reversing the effects of TGF $\beta$ signaling through the suppression of the SMAD-dependent canonical pathway and promotion of the non-canonical pathway. These effects were prevented by ALK5 overexpression. The inhibition of $\alpha$ - or $\beta$-adrenergic receptors alleviated the NE-mediated suppression of ALK5 expression. In conclusion, regional CSD protected rats from aortic aneurysm. NE inhibited SMAD2/3-dependent TGF $\beta$ signaling by suppressing ALK5 expression, which may serve an important
\end{abstract}

Correspondence to: $\mathrm{Dr}$ Zhiwei Wang, Department of Cardiovascular Surgery, Renmin Hospital of Wuhan University, 238 Jiefang Road, Wuhan, Hubei 430060, P.R. China

E-mail:wangzhiwei@whu.edu.cn

Key words: aortic remodeling, transforming growth factor $\beta$ signaling, norepinephrine, TGF $\beta$ type I receptor kinase, vascular smooth muscle cells, chemical sympathetic denervation role in VSMC biological functions. Both $\alpha$ - and $\beta$-adrenergic receptors were involved in the regulation of ALK5 expression by NE. Abnormal sympathetic innervation of the aorta may be used as a therapeutic target in aortic diseases.

\section{Introduction}

Aortic aneurysm, dissection and dilation share a common pathological feature of cystic medial necrosis and the histological characteristics of cystic medial necrosis include the loss of contractile vascular smooth muscle cells (VSMCs), leading to apoptosis and phenotypic switching, elastic fiber degradation and inflammatory cell infiltration (1). To date, the mechanism contributing to aortic remodeling remains unclear (2).

Previous studies have demonstrated that sympathetic activation and over-innervation promotes aortic dissection $(3,4)$ and that norepinephrine (NE) released by sympathetic nerve endings can upregulate the expression of matrix metallopeptidase-2 (MMP2) and promote aortic remodeling (5). However, the signaling pathway involved in NE regulation of aortic remodeling is still unknown.

Transforming growth factor (TGF) $\beta$ signaling serves a central role in aortic remodeling. Mutations of TGF $\beta$ family members such as TGFR-1/TGFR-2 result in a hereditary aortic aneurysm such as Loeys-Dietz syndrome (6). Marfan syndrome is a result of fibrillin-1 modification due to a defect or a mutation of the gene that encodes it, which is considered to regulate TGF $\beta$ bioavailability and activity by controlling access to, or the efficiency of, TGF $\beta$ activators (7). The dysregulation of the downstream TGF $\beta$ pathway signaling is also associated with aortic aneurysm (7). Since the homeostasis of TGF $\beta$ signaling is important in maintaining a normal structure of the aortic wall, the present study aimed to explore whether the sympathetic system may also regulate aortic remodeling via the TGF $\beta$ pathway.

Little is known regarding the effect of the sympathetic system on TGF $\beta$ signaling. Yang et al (8) investigated the interaction between the $\alpha_{1}$ adrenergic receptor and TGF $\beta$ type I receptor kinase (ALK5) pathways; however, the study was insufficient to clarify the relationship between the sympathetic system and TGF $\beta$ signaling. Therefore, the present study was designed to test a new hypothesis that the sympathetic system may regulate ALK5-mediated TGF $\beta$ signaling, thus 
serving a role in aortic remodeling. Previous studies have provided evidence on the use of ALK5 as a therapeutic target; for example, galunisertib, an ALK5 inhibitor, has antitumor activity in tumor-bearing animal models of breast, colon and lung cancers, and hepatocellular carcinoma (9); a phase II study has revealed that galunisertib treatment exerts hematologic improvements in low- and intermediate-risk myelodysplastic syndromes (10). Thus, the possibility of using ALK5 as a therapeutic target in aortic aneurysm was also explored in the present study.

\section{Materials and methods}

Animal experiments. As previously described (5), 50 male Sprague-Dawley rats (8 weeks, weight 267-299 g) were brought from ABLIII experimental animal laboratory of Wuhan university and housed in an animal room under controlled conditions of $20-26^{\circ} \mathrm{C}$ and $40-70 \%$ humidity on a $12 / 12-\mathrm{h}$ light/dark cycle. Normal chow was supplied to the control group, where as $0.25 \% \beta$-aminopropionitrile (BAPN) chow was supplied to the angiotensin II (AngII) and BAPN group to loosen the cross-link among elastic fibers (11-13). Chemical sympathetic denervation (CSD) was performed under pentobarbital anesthesia $(1 \% ; 30 \mathrm{mg} / \mathrm{kg})$ through a left paraspinal chest incision. The descending aorta between the left subclavian artery and the diaphragm was dissected and covered by a gauze pre-soaked in $20 \mu \mathrm{g} / \mu \mathrm{l}$ guanethidine for $30 \mathrm{~min}$. An osmotic minipump (Alzet, Durect Corp.) was implanted into the peritoneal cavity to infuse $1,000 \mathrm{ng} / \mathrm{kg} / \mathrm{min}$ AngII continuously for 4 weeks. The same operation and osmotic minipump was used in the control group where saline was used instead of guanethidine or AngII. At the end of 4 weeks, all surviving mice were sacrificed by $\mathrm{CO}_{2}\left(100 \% \mathrm{CO}_{2}, 2.5\right.$ liters per min, $5 \mathrm{~min}$ ) and survival rate was calculated as survived/total. The experiments were approved by The Ethics Committee of Renmin Hospital (Wuhan, China).

Cell culture and treatment. Mouse VSMC cell line (MOVAS) was obtained from ATCC and cultured in DMEM (Procell Life Science \& Technology Co., Ltd.) containing 10\% FBS (Procell Life Science \& Technology Co., Ltd.) at $37^{\circ} \mathrm{C}$ with $5 \% \mathrm{CO}_{2}$ and $95 \%$ air. The cells were sub-cultured to $70 \%$ confluence and subsequently cultured in DMEM without serum for $12 \mathrm{~h}$ before treatment; $1 \%$ FBS was added to the medium during any treatment.

ALK5 overexpression. Mouse ALK5 coding sequence was cloned into a pcw107 (V5) vector (Hanbio Biotechnology Co., Ltd.). A lentivirus was obtained using the PPMD2.G (Hanbio Biotechnology Co., Ltd.) and psPAX2 vectors (Hanbio Biotechnology Co., Ltd.) in 293T cells (China Center for Type Culture Collection). The lentivirus was aliquoted and transfected to the mouse VSMCs at the unified concentration using polybrene ( $8 \mu \mathrm{g} / \mathrm{ml}$, Sigma-Aldrich; Merck KGaA) for $72 \mathrm{~h}$.

Histology and immunostaining. Histology and immunostaining were performed as previously described (14). Briefly, sections were cut at $4 \mu \mathrm{m}$ from the paraffin-embedded aortic specimens of the rat model or control. The sections were stained with hematoxylin and eosin or elastica Van Gieson staining and immunostained with antibodies against each target protein (TH; 1:100, CST Biological Reagents Co., Ltd.; cat. no. 58844S; ALK5; 1:200, Abcam cat. no. ab31013). For the cell staining, having been seeded on the slides for $24 \mathrm{~h}$, the cells $\left(\sim 10^{5}\right.$ cells $\left./ \mathrm{cm}^{2}\right)$ were fixed in $4 \%$ paraformaldehyde (MACKLIN, China, Cat No. 30525-89-4) at $4^{\circ} \mathrm{C}$ for $20 \mathrm{~min}$ and stained using the same antibodies (incubated overnight at $4^{\circ} \mathrm{C}$ ) as above. An Olympus BX53 fluorescent microscope (Olympus Corporation) was used to investigate and capture images. Sympathetic nerve densities were determined by Image-Pro Plus 6.0 (Media Cybernetics, Inc.,) in tyrosine hydroxylase (TH) staining slides as previously described (3). Nerve density was calculated as the nerve area divided by the total area examined $\left(\mu \mathrm{m}^{2} / \mathrm{mm}^{2}\right)$.

Realtime quantitative PCR (RT-qPCR). Total RNA was extracted from cells (MOVAS) using RNAiso plus (Takara Bio, Inc.) according to the manufacturer's instructions followed by reverse transcription (SMART MMLV cDNA synthesis kit, Takara Biotechnology Co., Ltd.). A total of $20 \mu \mathrm{l}$ was used $(2 \mu \mathrm{l}$ cDNA, $10 \mu 1 \mathrm{SYBR}^{\circledR}$ Green (Thermo Fisher Scientific, Inc.), $2 \mu 1$ primer (Servicebio), $6 \mu 1$ water) on an ABI $9700 \mathrm{qPCR}$ machine (Applied Biosystems; Thermo Fisher Scientific, Inc.). The settings were: $93^{\circ} \mathrm{C}$ for $40 \mathrm{sec}, 58^{\circ} \mathrm{C}$ for $30 \mathrm{sec}$ and $72^{\circ} \mathrm{C}$ for $60 \mathrm{sec}$ (35 cycles). The primers are listed in Table I. The result was calculated by the $2^{-\Delta \Delta \mathrm{Cq}}$ method (15).

Western blotting. RIPA lysis buffer (Beyotime Biotechnology, Inc. cat no. P0013B) was used to extract total proteins from cells and bicinchoninic acid assay was used to measure the protein concentration. Protein (20-30 $\mu \mathrm{g})$ was loaded onto a $15 \%$ SDS-PAGE gel and ran at $100 \mathrm{~V}$. Then the protein was transferred onto PVDF membranes and blocked by $5 \%$ milk at room temperature for $1 \mathrm{~h}$. Primary antibodies were incubated with the membrane overnight at $4^{\circ} \mathrm{C}$ : ALK5, Abcam, cat. no. ab31013, 1:1,000; SMAD2/3, CST Biological Reagents Co., Ltd., cat. no. 5678, 1:2,000; p-SAMD2/3, Santa Cruz Biotechnology, Inc., cat. no. sc-11769,1:200; ERK1/2, Biorbyt Technology, Inc., cat. no. orb216186, 1:500; p-ERK1/2, CST Biological Reagents Co., Ltd., cat. no. 8544, 1:1,000; JNK1/2, Santa Cruz Biotechnology, Inc., cat. no. sc-7345, 1:200; p-JNK, Invitrogen (Thermo Fisher Scientific, Inc.), cat. no. 700031, 1:1,000; GAPDH, Santa Cruz Biotechnology, Inc., cat. no. sc47724, 1:1,000. The membrane was then incubated with the secondary antibodies and chemiluminescence method was used to acquire images. Image-Pro Plus 6.0 (Media Cybernetics, Inc.) was used to analysis the bands.

Cell migration assay. Cell migration assay was performed using mouse VSMCs. Cells were cultured in DMEM supplemented with $1 \%$ FBS as VSMCs stopped proliferating in $1 \%$ FBS (Fig. S1). The scratch was created with a $200 \mu$ l pipette tip and the scratch closure was monitored at 24 and $48 \mathrm{~h}$. Scratch closure was quantified using ImageJ 1.52t (National Institutes of Health).

NE concentration assay. Small segments of the aorta were immediately placed in $0.1 \mathrm{M}$ Perchloric acid ( $\mathrm{HClO} 4)$ solution after harvest and kept overnight and then stored under $-80^{\circ} \mathrm{C}$. The samples was ground and dissolved in the same volume 
Table I. Primers used in the present study.

\begin{tabular}{|c|c|}
\hline Target genes & Forward 5'-3' \\
\hline
\end{tabular}

Mouse ALK5

Mouse TGF $\beta 1$

Mouse INHBA

Mouse BMP4

\section{GAAAAGCAGTCAGCTGGCCTT \\ CTGCTGACCCCCACTGATAC \\ AAATCAGAACGCCTCCGCTA TCCGTCCCTGATGGGATTCT}

CTTCATTTGGCACACGGTGG AGCCCTGTATTCCGTCTCCT TCCCGAGTGTAGAGTTCGGT TGGTGTCTCATTGGTTCCTGC

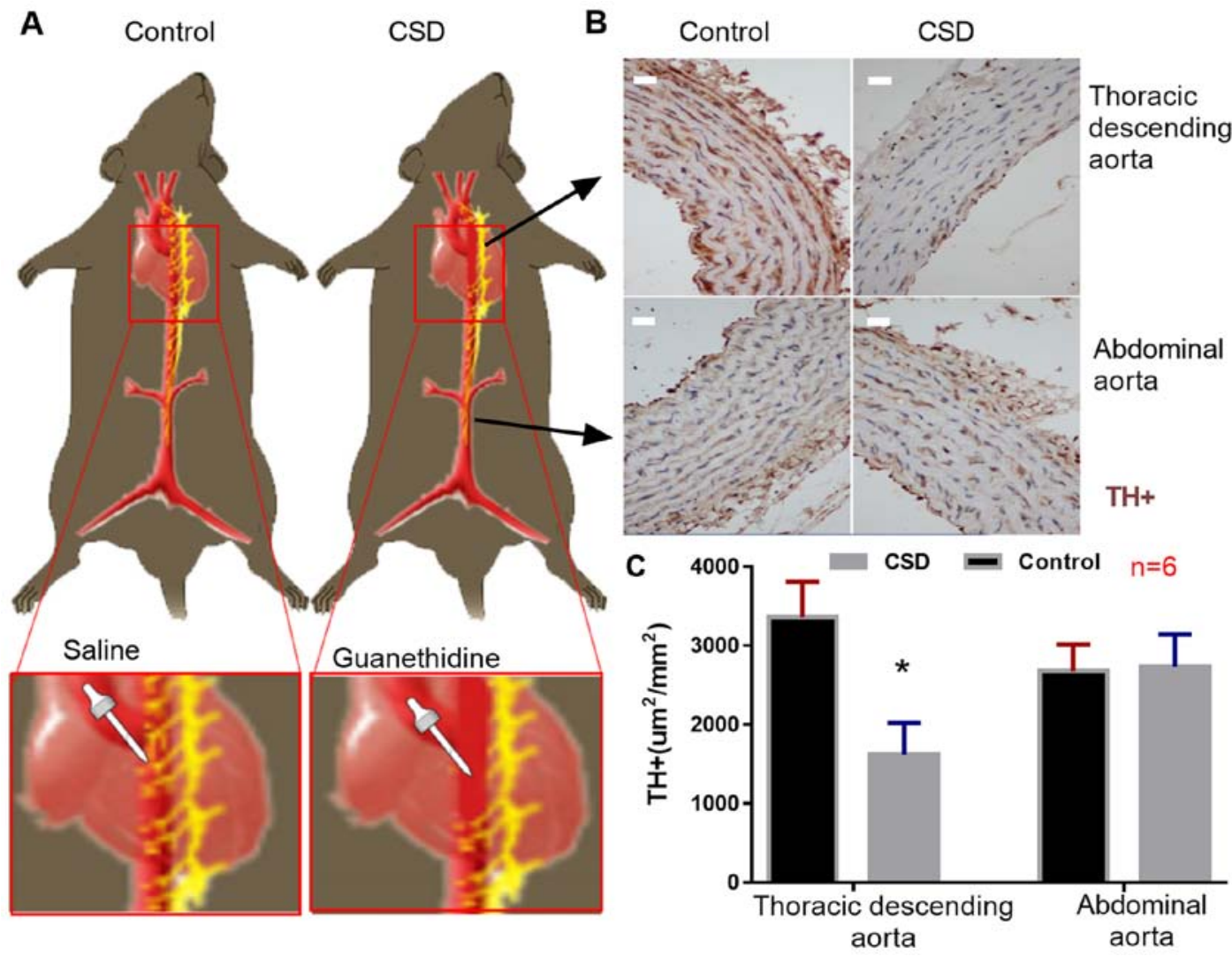

Figure 1. Schematic diagram and the results of CSD. (A) Schematic diagram: CSD was performed on descending aorta with guanethidine but not on abdominal aorta. Saline was used in the control group. (B and $\mathrm{C}$ ) $\mathrm{TH}^{+}$sympathetic nerve endings were stained. The number of $\mathrm{TH}^{+}$cells was significantly decreased after CSD in the thoracic descending aorta compared with the control group, whereas no change was observed in the abdominal aorta. Scale bars, $400 \mu \mathrm{m} ; \mathrm{n}=6$ in each group. " $\mathrm{P}<0.01$ vs. control. $\mathrm{CSD}$, chemical sympathetic denervation; $\mathrm{TH}^{+}$, tyrosine hydroxylase-positive.

of saline $(50 \mathrm{mg} / \mathrm{ml})$. The concentration of AngII and NE in the aorta were detected using ELISA kits (AngII, Cloud-Clone Corp. (CEA005Ra); NE, Eagle Biosciences, Inc. (SKU: NOR31-K01) according to the manufacturers' instructions.

Cell proliferation assay. Cell Counting Kit-8 (CCK8) proliferation assay kit was obtained from Biosharp Life Sciences (cat. no: BS350B) and used according to the manufacturer's protocol. Mouse VSMCs were seeded in 96-well plates at a density of $3 \times 10^{3}$ cells/well. After $24 \mathrm{~h}$, DMEM containing $1 \%$ FBS and $10 \mu \mathrm{l}$ CCK8 was added into each well and incubated for $4 \mathrm{~h}$ at $37^{\circ} \mathrm{C}$. The optical density was read at $450 \mathrm{~nm}$ using a Multiskan MK3 microplate reader (Thermo Fisher Scientific, Inc.). The NE and FBS concentration gradient experiment was performed with different dosages of NE or FBS in DMEM by cell counting. Briefly, MOVAS cells were passaged and synchronized in 1\% FBS for $12 \mathrm{~h}$ and 1, 10, 50, 100 and $200 \mathrm{~nm} \mathrm{NE}$ and
0, 1, 2, 5 and 10\% FBS DMEM was used to culture cells. Cell numbers were counted at 24,48 and $72 \mathrm{~h}$.

Apoptosis assay. DMEM containing 1\% FBS was applied to cells treated with NE, NE+ALK5 or PBS in each group. According to the manufacturer's instructions of the apoptosis assay kit (Nanjing KeyGen Biotech Co., Ltd.), after trypsinization, cells were washed twice, centrifuged $\left(800 \mathrm{x} \mathrm{g}, 4^{\circ} \mathrm{C}\right.$ for $5 \mathrm{~min}$ ) and resuspended in $500 \mu \mathrm{l}$ Binding Buffer from the kit). Next, the suspension was mixed with $5 \mu$ l AnnexinV-FITC, and $5 \mu 1$ propidium iodide (PI) was added. After incubation in the dark for 5-15 min, flow cytometry assay was performed using a CytoFLEX flow cytometer with Cytexpert software (Beckman Coulter, Inc.; version 2.3). The negative control was without AnnexinV-FITC and PI. Late apoptosis was assessed and the most significant apoptotic group was used as a positive control, as suggested by the kit manufacturer. 

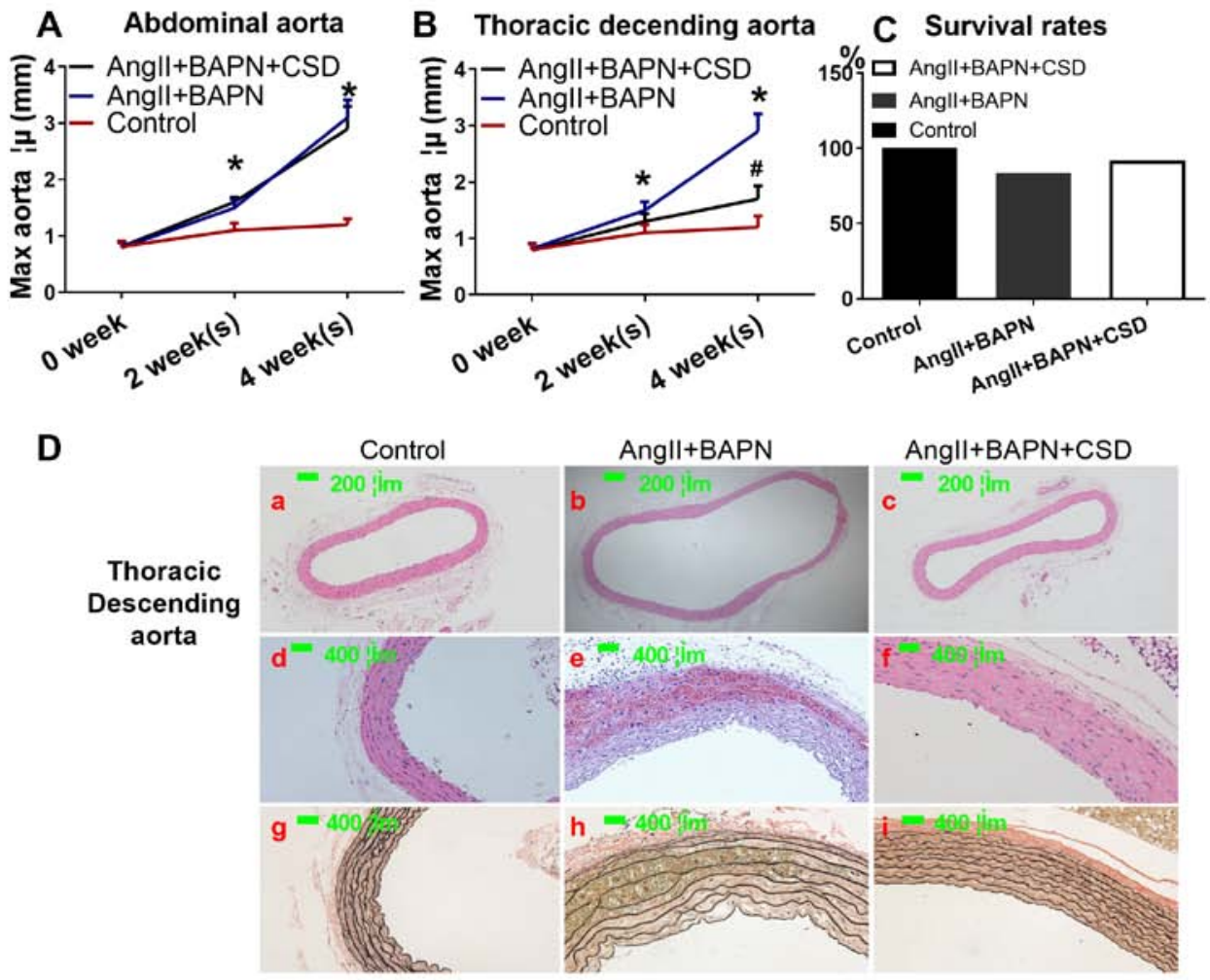

Angll+BAPN+CSD

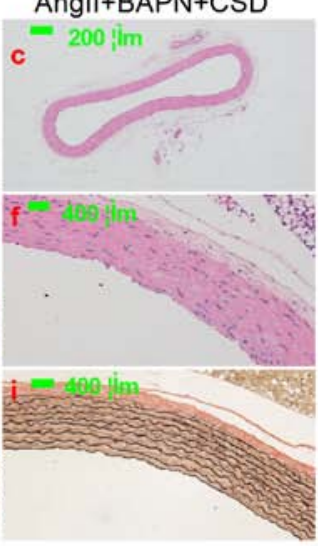

E

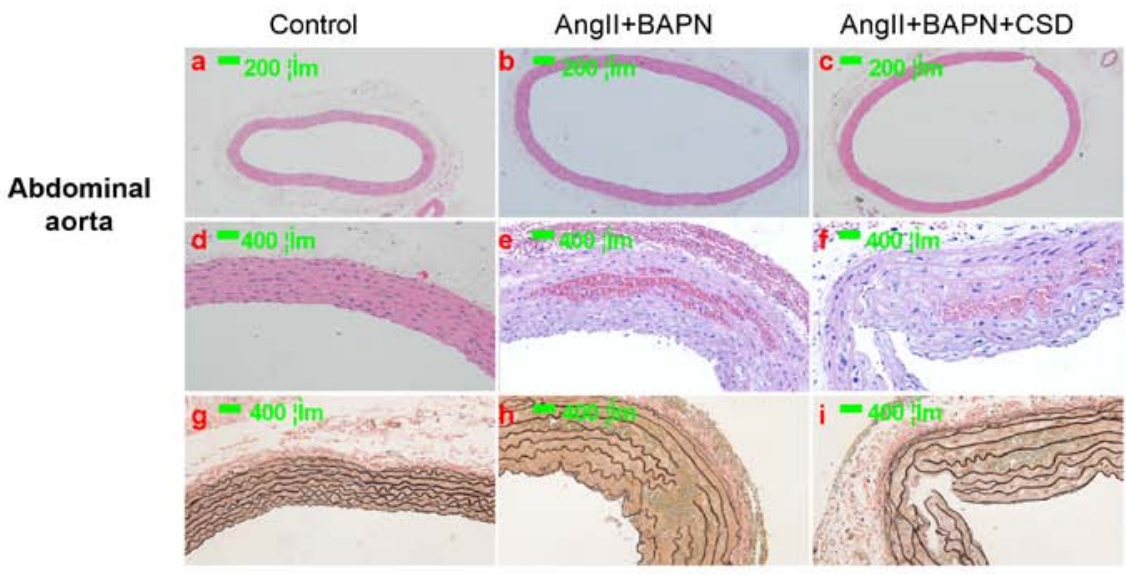

Figure 2. Impact of CSD on aorta dilation and aortic remodeling induced by AngII+BAPN. (A and B) AngII+BAPN induced significant aortic dilation in the thoracic descending and the abdominal aortas. When CSD was performed, the AngII+BAPN-induced aortic dilation was alleviated in (B) the thoracic descending aorta, but not in (A) the abdominal aorta. (C) The survival rate of rats in each group was not significantly different. (D and E) HE and elastica Van Gieson staining demonstrated significant aortic dilation and elastic fiber destruction, which was rescued by CSD in (D) the thoracic descending aorta, but not in (E) the abdominal aorta. $\mathrm{n}=12$ in each group. ${ }^{*} \mathrm{P}<0.01$ vs. control. ${ }^{*} \mathrm{P}<0.01$ vs. AngII+BAPN group. AngII, angiotensin II; BAPN, $\beta$-aminopropionitrile; $\mathrm{CSD}$, chemical sympathetic denervation; HE hematoxylin and eosin.

Statistical analysis. All quantitative data are presented as mean \pm standard deviation. Statistical analysis was performed using GraphPad Prism software (version 6; GraphPad Software Inc.). Unpaired Student's t-test was performed to calculate the differences between two groups, and one-way ANOVA was performed when three groups were compared. Tukey's post hoc test was used for multiple comparisons. $\mathrm{P}<0.01$ was considered to indicate a statistically significant difference.

\section{Results}

Aortic CSD protects AngII-induced aortic remodeling. At the end of the animal experiment, $\mathrm{TH}$-positive $\left(\mathrm{TH}^{+}\right)$ cells (sympathetic nerve endings) were immunostained. The number of $\mathrm{TH}^{+}$cells were significantly decreased in the descending aortas, but not in the abdominal aortas of the CSD group compared with those in the control groups (Fig. 1). No aortic rupture was observed in the control group, but two (16.7\%) ruptures in the AngII+BAPN group and one in the AngII+BAPN+CSD group (8.3\%) were present (Fig. 2C). The diameter of both the descending and abdominal aorta increased with time in the AngII- and BAPN-treated groups (Fig. 2A, B, D-a, D-b, E-a and E-b). CSD rescued the aortic dilation in the descending, but not in the abdominal aorta (Fig. 2A, B, D-c and E-c). AngII and BAPN induced intramural aortic hematoma and elastic fiber destruction, 
A

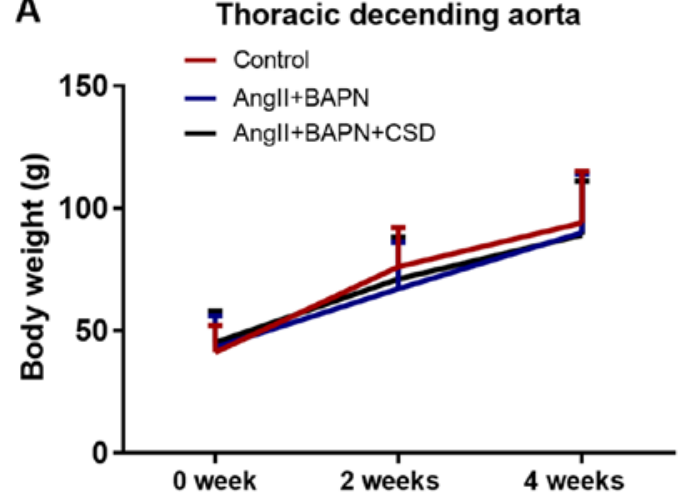

C

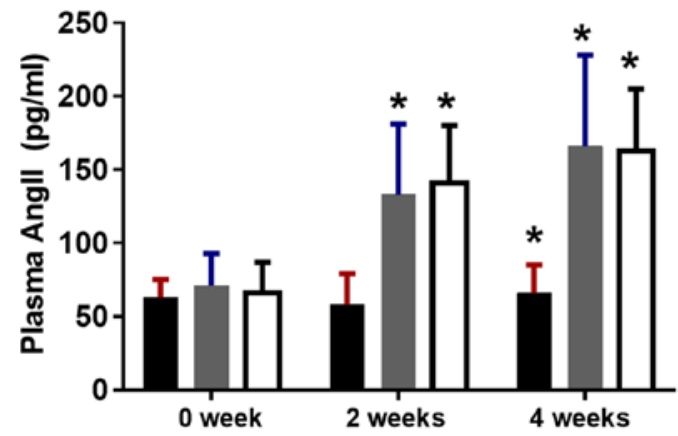

E

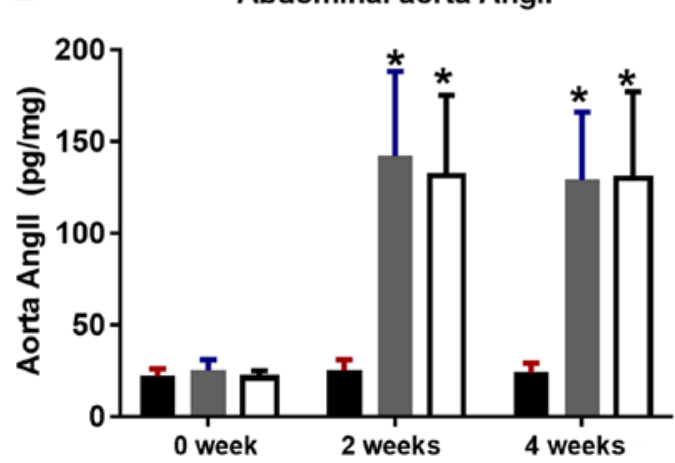

G

G Abdominal aorta NE

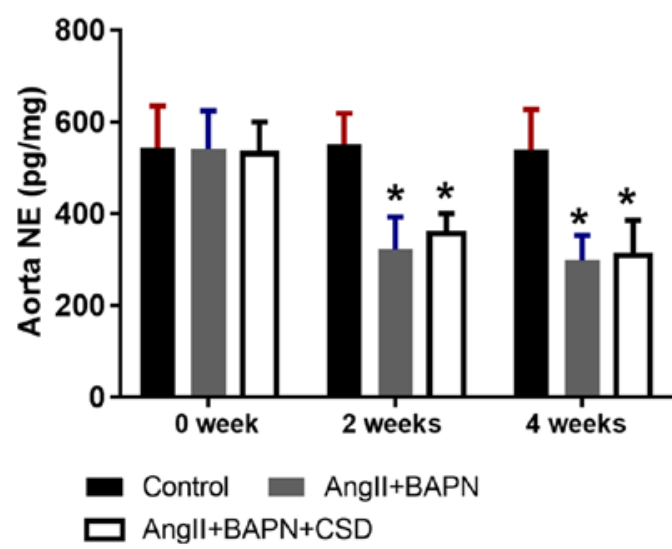

B

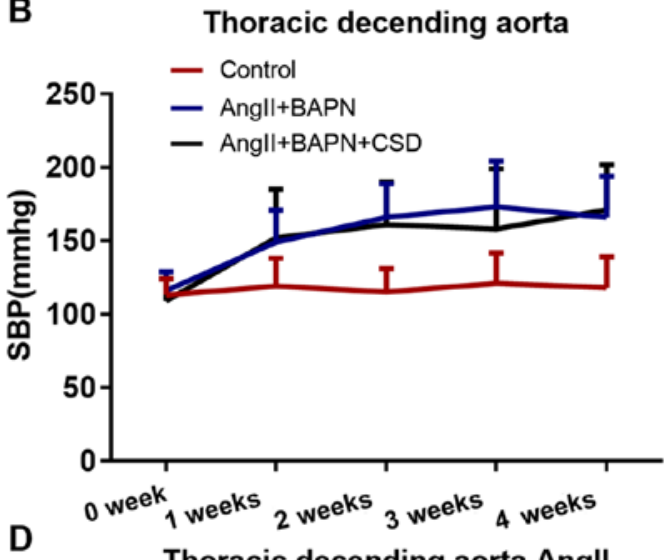

Thoracic decending aorta Angll

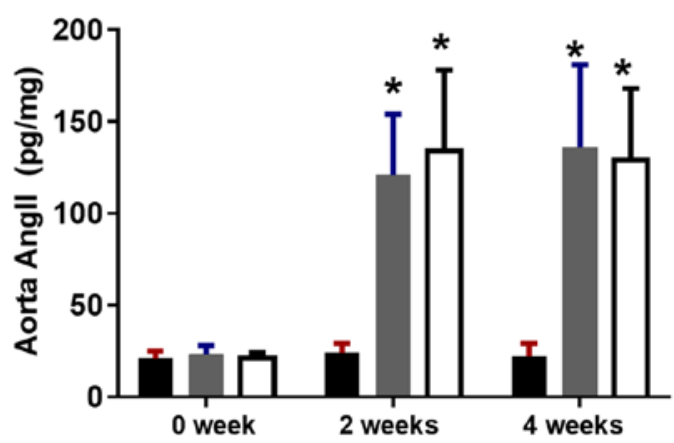

F

Thoracic decending aorta NE

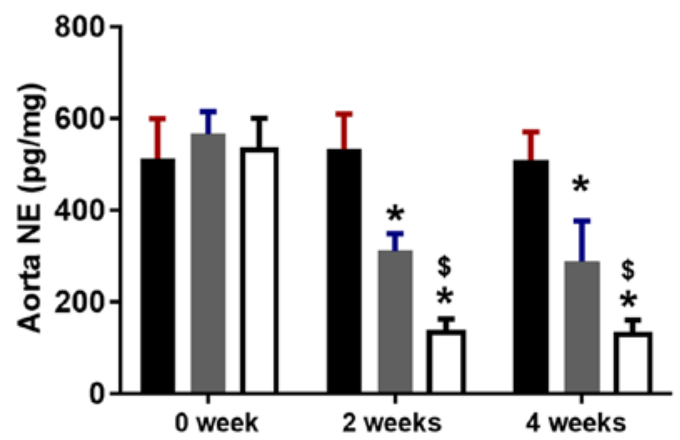

H

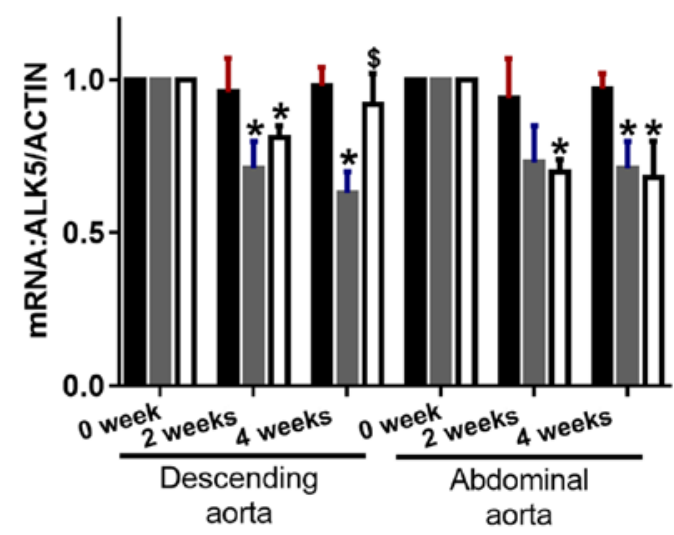

Figure 3. Effects of CSD on AngII-induced NE release and ALK5 expression. (A) Rat body weight was similar among all groups. (B) SBP was higher in the AngII-treated groups. CSD had no significant effect on SBP. (C) Plasma and (D and E) aortic tissue concentration of AngII in (D) the thoracic descending and (E) the abdominal aorta was higher in the AngII-treated groups compared with those in the control group. CSD had no significant effects on AngII concentration. (F and G) Tissue NE concentration in (F) the thoracic descending aorta and $(\mathrm{G})$ the abdominal aorta was lower in the AngII-treated groups compared with the control group. CSD further decreased NE in (F) the thoracic descending aorta, but not in (G) the abdominal aorta. (H) ALK5 expression in the thoracic descending and the abdominal aortas was downregulated in the AngII-treated groups. CSD further downregulated ALK5 in the thoracic descending aorta, but not in the abdominal aorta. ${ }^{*} \mathrm{P}<0.01$ vs. control; ${ }^{\$} \mathrm{P}<0.01$ vs. AngII+BAPN. SBP, systolic blood pressure; AngII, angiotensin II; BAPN, $\beta$-aminopropionitrile; ALK5, transforming growth factor $\beta$ type I receptor kinase; NE, norepinephrine. 

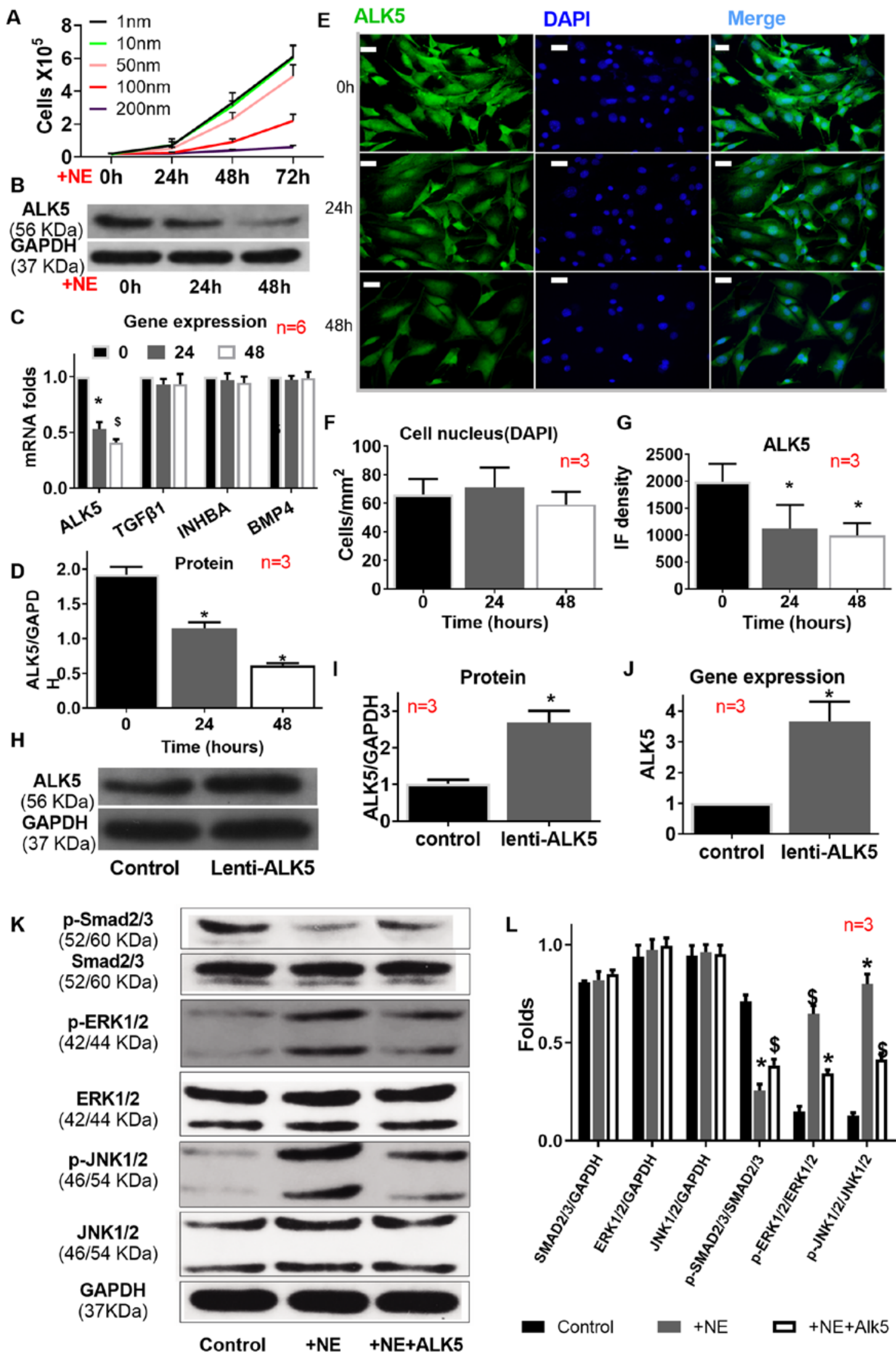

Figure 4. Impact of ALK5 overexpression on ALK5 expression and signaling transduction. (A) NE (100 nm) significantly decreased VSMC proliferation and suppressed ALK5 expression, as indicated by (B-D) western blotting (C) reverse transcription-quantitative PCR and (E-G) immunofluorescence, but (C) did not affect TGF $\beta 1$, INHBA or BMP4 expression. (H-J) ALK5 was successfully overexpressed.(K and L) NE suppressed SMAD2/3 signaling, but activated ERK1/2 and JNK1/2 signaling. ALK5 overexpression partially rescued the effect of NE. Scale bar $=400 \mu \mathrm{m}$. " $\mathrm{P}<0.01 \mathrm{vs}$. control; ${ }^{\$} \mathrm{P}<0.01 \mathrm{vs}$. AngII+BAPN. NE, norepinephrine; ALK5, transforming growth factor $\beta$ type I receptor kinase; TGF, transforming growth factor; AngII, angiotensin II; BAPN, $\beta$-aminopropionitrile; p, phosphorylated; IF, immunofluorescence; INHBA, inhibin subunit $\beta$ A; BMP4, bone morphogenetic protein 4. 

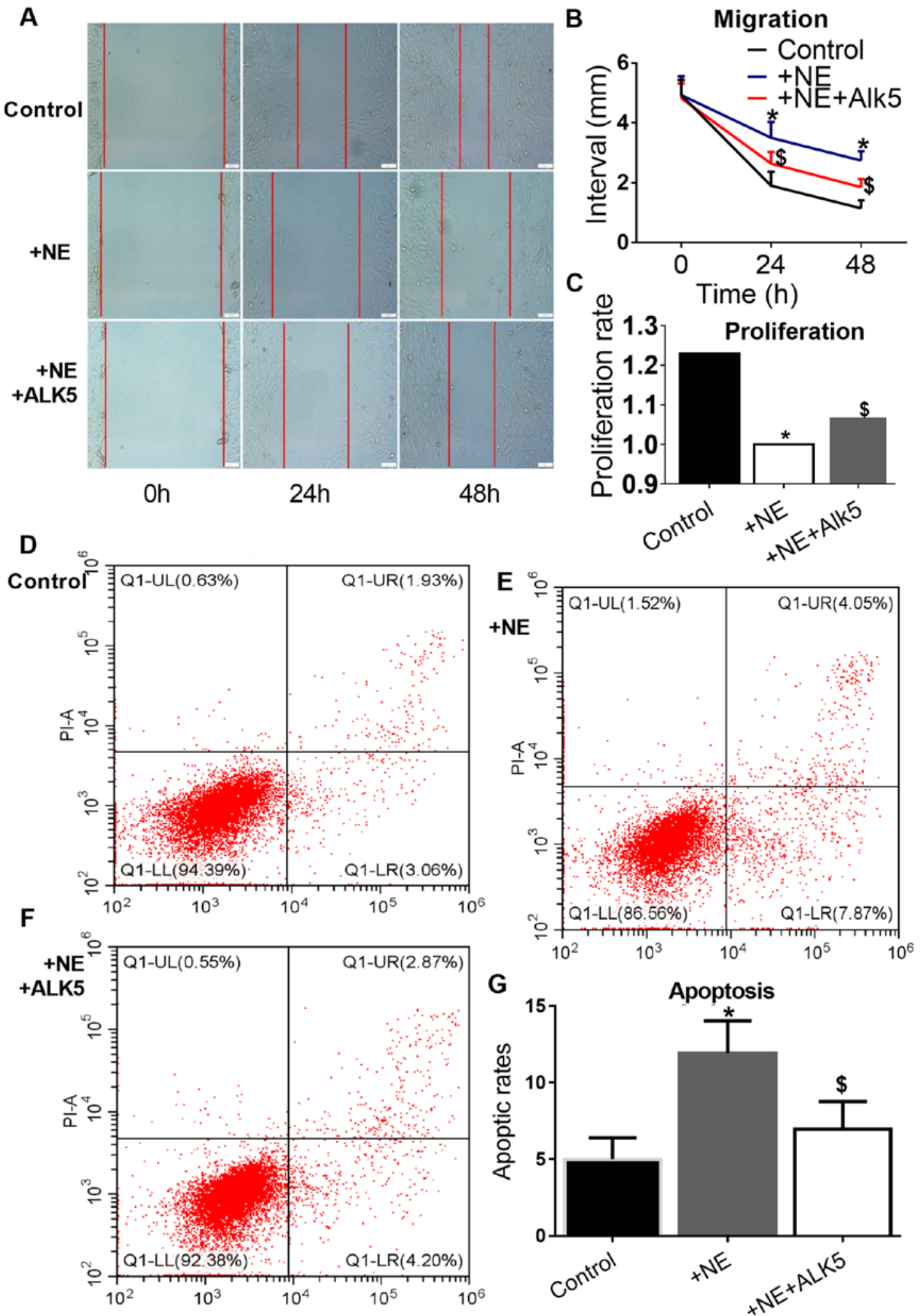

Figure 5. Effect of NE and ALK5 overexpression on VSMCs migration, proliferation, and apoptosis. NE inhibits VSMCs (A and B) migration and (C) proliferation, but (D-G) promotes apoptosis. ALK5 overexpression partially rescued the effects of NE. Scale bars $=400 \mu \mathrm{m}$. " $\mathrm{P}<0.01 \mathrm{vs}$. control; ${ }^{\$} \mathrm{P}<0.01 \mathrm{vs}$ AngII+BAPN. VSMCs, vascular smooth muscle cells; ALK5, transforming growth factor $\beta$ type I receptor kinase; NE, norepinephrine; AngII, angiotensin II; BAPN, $\beta$-aminopropionitrile; PI, propidium iodide.

which was not observed in the control group. In addition, CSD rescued intramural aortic hematoma and elastic fiber destruction in the descending, but not in the abdominal aorta (Fig. 2D-d-i and E-d-i).
CSD alleviates AngII-induced NE release and ALK5 downregulation in the aorta. No significant difference in body weight was observed among the treatment groups (Fig. 3A). AngII increased the systemic blood pressure compared with 
the control group, and regional CSD did not alleviate the AngII-induced hypertension (Fig. 3B). In the AngII-pumped groups, the concentrations of AngII in the plasma, the thoracic descending and the abdominal aorta significantly increased compared with those in the control group, and regional CSD did not affect AngII concentration in the plasma and aortic tissue (Fig. 3C-E). AngII also reduced the NE concentration in the thoracic descending and abdominal aortas of the AngII-pumped groups compared with the control group. In the CSD segment, the NE concentration in the thoracic descending aorta was lower compared with that in the aorta of the AngII+BAPN group, and this difference was not observed in the abdominal aorta (Fig. 3F and G). ALK5 expression was downregulated in the aortas of the rats in the AngII+BAPN group compared with those in the control group, and CSD rescued this effect in the thoracic descending aorta (significant at the end of 4 weeks), but not in the abdominal aorta (Fig. 3H).

NE modulates TGF $\beta$ signaling by suppressing ALK5 expression in VSMCs. Based on the concentration gradient experiment, $100 \mathrm{nM}$ NE was used to treat mouse VSMCs. Compared with the control group the expression of ALK5 was significantly downregulated at $24 \mathrm{~h}$ after NE treatment was further downregulated at $48 \mathrm{~h}$ according to the results obtained by western blotting (Fig. 4B and C), qPCR (Fig. 4D), and immunofluorescence (Fig. 4E-I); however the expression of the TGF $\beta$ receptor ligands; TGF $\beta 1$, Inhibin Subunit $\beta$ A (INHBA) and Bone Morphogenetic Protein 4 (BMP4) as determined by qPCR did not change (Fig. 4D). NE treatment also altered the dominance of TGF $\beta$ signaling, as it suppressed the phosphorylation of SMAD2/3 and promoted the phosphorylation of ERK1/2 and JNK1/2, which was partially reversed by ALK5 overexpression (Fig. 4K and L).

ALK5 overexpression reverses the effects of NE on VSMC proliferation, migration and apoptosis. CCK8 assay was used to investigate the effects of $100 \mathrm{nM} \mathrm{NE}$ on VSMC proliferation. A significant inhibition of mouse VSMC proliferation and migration in the wound healing assay by NE was observed. In addition, a promoting effect of NE on VSMC apoptosis was observed. However, the effect of NE on VSMC proliferation, migration and apoptosis was reversed by ALK5 overexpression (Fig. 5).

$\alpha$ - and $\beta$-adrenergic receptors are involved in NE-ALK5 signaling. Among the adrenoceptors, $\alpha_{1}$ and $\beta_{1}$ receptors are very important in maintaining artery structure homeostasis $(16,17)$. To clarify which adrenergic receptors were involved in NE-ALK5 signaling, selective receptor antagonists $10 \mu \mathrm{M}$ prazosin and metoprolol were used to block $\alpha_{1^{-}}$or $\beta_{1}$-adrenergic receptors, respectively. Both prazosin and metoprolol partially reversed the inhibition of NE on ALK5 expression, with metoprolol exerting a slightly stronger effect (Fig. 6).

\section{Discussion}

A number of studies have focused on the regulation of the vessel tone and blood pressure by the sympathetic system, but have neglected its impact on aortic wall structure $(18,19)$. Evidence

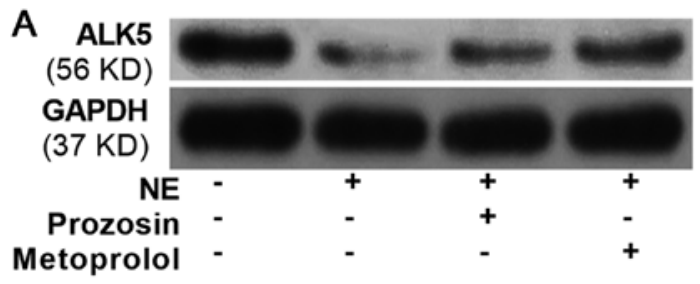

B

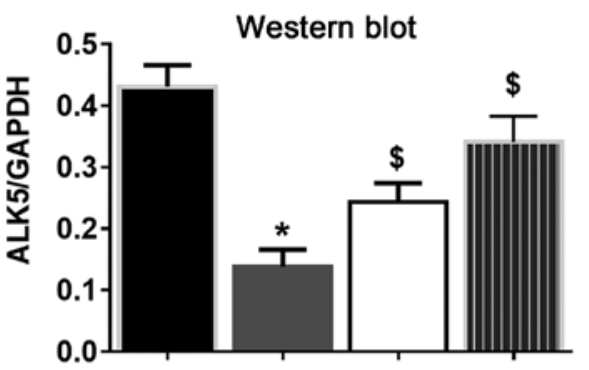<smiles>[CH][CH]</smiles>

C

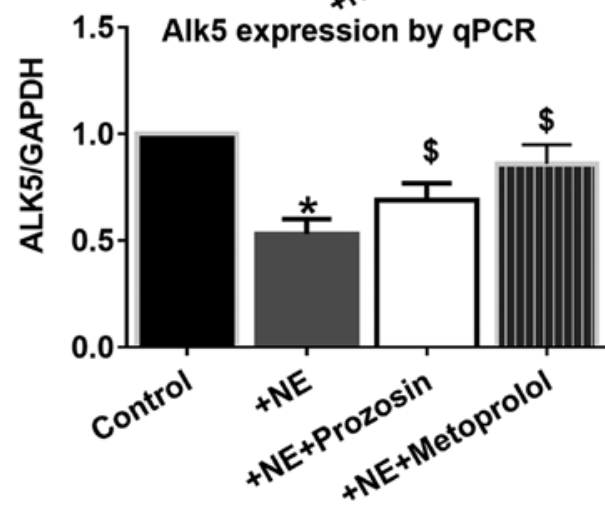

Figure 6. Involvement of $\alpha_{1}$ and $\beta_{1}$ adrenergic receptor in the modulation of NE on ALK5 expression. The inhibitory effects of NE on ALK5 expression are partially blocked by $\alpha_{1}$ and $\beta_{1}$ adrenergic receptor inhibitors, prozosin and metprolol, respectively, indicated by (A and B) western blot and (C) qPCR. ${ }^{*} \mathrm{P}<0.01$ vs. control; ${ }^{\$} \mathrm{P}<0.01$ vs. $+\mathrm{NE}$. ALK5, transforming growth factor $\beta$ type I receptor kinase; NE, norepinephrine.

indicates that the sympathetic system not only regulates the arteries from a functional aspect, but also from a structural aspect. First, there is a clear difference in the sympathetic innervation of arteries and veins; sympathetic innervation is rich in arteries but poor in veins (20). Furthermore, sympathetic innervation increases during development (21). Chronic hypoxia is a risk factor of aortic $(22-24)$ and small artery $(25,26)$ diseases, as well as an inducer of arterial sympathetic innervation (27). Thus, a regional CSD method was used in the present study to investigate the role of aortic sympathetic innervation. Compared with global sympathetic denervation or surgical aortic sympathetic denervation (thoracic sympathectomy), this method is less likely to affect systemic blood pressure (28). Blood pressure is a strong factor promoting aortic remodeling (29). The histological and pathological features of the descending (CSD segment) and abdominal aorta (non-CSD segment) were compared in order to exclude the possibility that CSD may also affect the non-CSD area. In the descending aorta, the number of $\mathrm{TH}^{+}$cells (sympathetic nerve endings) was significantly decreased, and the intramural aortic hematoma and elastic fiber destruction were rescued compared with the 


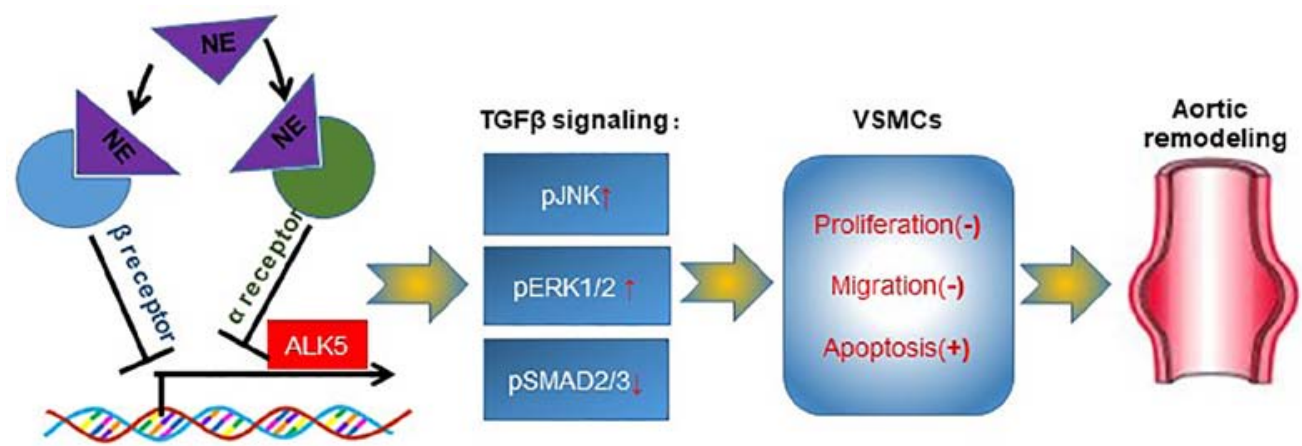

Figure 7. Schematic diagram of the new findings of the present study. NE, norepinephrine; TGF, transforming growth factor; VSMCs, vascular smooth muscle cells; ALK5, transforming growth factor $\beta$ type I receptor kinase.

control group. By contrast, in the abdominal aorta (non-CSD segment), the number of $\mathrm{TH}^{+}$cells was not decreased, and the intramural aortic hematoma and elastic fiber were not rescued. These results indicated that regional CSD was only effective in the treated region.

The present study identified ALK5 as a target of the sympathetic nervous system. NE suppressed TGF $\beta$ receptor1 expression without any impact on the TGF $\beta$ receptor ligands (although the expression of TGF $\beta$ receptor ligands was only tested by qPCR, not western blotting). To date, only a limited number of studies have explored whether and how the autonomic nervous system regulates ALK5 expression and signaling. In a rat cerebral ischemia/reperfusion model, Ma et al (30) have demonstrated that stimulation of the vagus nerve regulates Growth Differentiation Factor 11 (GDF11) and ALK5 expression, and hypothesized that GDF11/ALK5 may represent a potential target for stroke therapy. Neuropilins1, an axon elongation inducer, inhibits the expression of both ALK1 and ALK5 (31). Yang et al (8) demonstrated that $\alpha_{1}$-adrenergic receptor/ALK5 interaction contributes to doxazosin-induced apoptosis, which is further enhanced by TGF $\beta 1$ in association with attenuating SMAD3 phosphorylation in H9C2 cells. By contrast, the results of the present study demonstrated that both $\alpha$ - and $\beta$-adrenergic receptors were involved in the regulation of NE on ALK5 expression. The present study and the aforementioned previous studies further confirmed the interaction between TGF $\beta$ signaling and the autonomic nervous system.

ALK5 is a membrane-bound receptor protein of the TGF $\beta$ superfamily of signaling ligands (32). When bound to TGF $\beta$, ALK5 transduces the TGF $\beta$ signal from the cell surface to the cytoplasm. Abnormal expression and/or activation of ALK5 induce changes in the downstream signaling transduction and diseases (30). Mutations in the ALK5 gene are associated with the Loeys-Dietz aortic aneurysm syndrome (6). The present study revealed that postnatal modulation of ALK5 by a sympathetic transmitter also contributes to aortic remodeling.

TGF $\beta$ signaling consists of SMAD2/3-dependent and non-SMAD2/3-dependent cascades; the former is termed canonical TGF $\beta$ signaling, and the latter is termed non-canonical TGF $\beta$ signaling $(33,34)$. Inhibition of the non-canonical TGF $\beta$ signaling molecules such as ERK1/2 and/or JNK1 may rescue aortic aneurysms $(33,35,36)$. The results of the present study suggested that ALK5 repression by NE also induced TGF $\beta$ signaling dominance switch and aortic remodeling. This indicated a strong impact of NE and the sympathetic nervous system on ALK5 expression and downstream signaling. TGF $\beta$ signaling serves broad biological functions (34). Its normal status is essential in the development and maintenance of the physiological balance, but when disturbed, may result in a number of diseases (34). Thus, it is possible that $\mathrm{NE}$ and the sympathetic nervous system may also exert important roles in other diseases, such as arterial diseases, via modulating ALK5 expression and TGF $\beta$ signaling.

A possible limitation of the present study was the NE tissue concentration. Our previous study has demonstrated that AngII promotes NE release from the sympathetic nerve endings (5). Subsequently, cells in the tissue (such as VSMCs) interact with more NE, and the NE in the extracellular fluid is washed away by the blood flow. Thus, more NE release results in lower tissue NE concentration (33). CSD also reduces tissue $\mathrm{NE}$ by reducing the number of sympathetic nerve endings and NE release, resulting in less NE acting on cells (VSMCs and others). This is consistent with another study (37). The mortality rate in the present study was lower compared with some studies $(12,38)$ as older rats were used in the present study instead of 3-week-old rats. Tatsuo Kawai et al (11) used a similar protocol in modeling abdominal aortic aneurysm and observed similar mortality.

The biological function of the sympathetic nervous system is broad. NE and ALK5 may not be the only molecules in the sympathetic nervous system to regulate TGF $\beta$ signaling, and TGF $\beta$ signaling modulation may not be the only mechanism of the sympathetic nervous system to serve a role in aortic disease pathogenesis. However, based on previous and the present research, the sympathetic nervous system, or even other autonomic nervous systems, such as the vagal nerve system, may serve as a therapeutic target to combat aortic diseases. Whether directly inhibiting ALK5 in control cells without NE treatment also produces the same results requires further research. Although this was a limitation of the present study, it did not affect the conclusions as previous studies have demonstrated similar findings on the role of ALK5 in VSMC proliferation and migration $(32,35)$.

The present study confirmed the important role of sympathetic system dysregulation in aortic remodeling. In addition, the present study also revealed a new signaling pathway regulating the sympathetic-adrenergic system. This pathway and its in vivo function have not been previously investigated. 
In conclusion, the results of the present study demonstrated that regional CSD protected the rats from aortic aneurysm. The sympathetic transmitter NE modulated TGF $\beta$ signaling by suppressing ALK5 expression, serving an important role in the biological functions of VSMCs. Both $\alpha$ - and $\beta$-adrenergic receptors may be involved in the regulation of NE on ALK5 expression. Thus, abnormal sympathetic innervation of the aorta may serve as a therapeutic target in aortic diseases (Fig. 7).

\section{Acknowledgements}

The authors thank Dr Junmou Hong from the Zhongshan Hospital at Xiamen University for his valuable advice to the present study.

\section{Funding}

This study was supported by The National Natural Science Foundation of China (grant no. 81600367 to Zhipeng Hu).

\section{Availability of data and materials}

The datasets used and/or analyzed during the current study are available from the corresponding author on reasonable request.

\section{Authors' contributions}

$\mathrm{ZW}$ and $\mathrm{ZH}$ conceived the hypothesis and designed the study protocol. $\mathrm{ZH}$ wrote the manuscript. $\mathrm{BL}, \mathrm{RC}, \mathrm{QW}$ and $\mathrm{ZH}$ performed most of the experiments. $\mathrm{XH}, \mathrm{MZ}$, and FJ participated in some of the experiments. All authors read and approved the final manuscript.

\section{Ethics approval and consent to participate}

Animal experiments were approved by the ethics committee of Renmin hospital, Wuhan University (Wuhan, China).

\section{Patient consent for publication}

Not applicable.

\section{Competing interests}

The authors declare that they have no competing interests.

\section{References}

1. Akutsu K, Kawamoto M, Sato N, Yamamoto T, Tamura K Mizuno K and Tanaka K: Acute aortic dissection associated with cystic medial necrosis of unknown etiology. J Nippon Med Sch 79: 159-162, 2012.

2. Fraga-Silva RA and Trachet B: Editorial: Novel insights on aortic aneurysm. Curr Pharm Des 21: 3993-3995, 2015.

3. Zhipeng H, Zhiwei W, Lilei Y, Hao Z, Hongbing W, Zongli R, Hao $\mathrm{C}$ and Xiaoping H: Sympathetic hyperactivity and aortic sympathetic nerve sprouting in patients with thoracic aortic dissection. Ann Vasc Surg 28: 1243-1248, 2014.

4. Hu R, Wang Z, Ren Z and Liu M: Autonomic remodeling may be responsible for decreased incidence of aortic dissection in STZ-induced diabetic rats via down-regulation of matrix metalloprotease 2. BMC Cardiovasc Disord 16: 200, 2016.
5. Hu Z, Wang Z, Wu H, Yang Z, Jiang W, Li L and Hu X: Ang II enhances noradrenaline release from sympathetic nerve endings thus contributing to the up-regulation of metalloprotease- 2 in aortic dissection patients' aorta wall. PLoS One 8: e76922, 2013.

6. Loeys BL, Schwarze U, Holm T, Callewaert BL, Thomas GH, Pannu H, De Backer JF, Oswald GL, Symoens S, Manouvrier S, et al: Aneurysm syndromes caused by mutations in the TGF-beta receptor. N Engl J Med 355: 788-798, 2006.

7. Lindsay ME and Dietz HC: Lessons on the pathogenesis of aneurysm from heritable conditions. Nature 473: 308-316, 2011.

8. Yang YF, Wu CC, Chen WP and Su MJ: Transforming growth factor-beta type I receptor/ALK 5 contributes to doxazosin-induced apoptosis in H9C2 cells. Naunyn Schmiedebergs Arch Pharmacol 380: 561-567, 2009.

9. Herbertz S, Sawyer JS, Stauber AJ, Gueorguieva I, Driscoll KE, Estrem ST, Cleverly AL, Desaiah D, Guba SC, Benhadji KA, et al: Clinical development of galunisertib (LY2157299 monohydrate), a small molecule inhibitor of transforming growth factor-beta signaling pathway. Drug Des Devel Ther 9: 4479-4499, 2015.

10. Santini V, Valcárcel D, Platzbecker U, Komrokji RS, Cleverly AL, Lahn MM, Janssen J, Zhao Y, Chiang A, Giagounidis A, et al: Phase II study of the ALK5 inhibitor galunisertib in very low-, low-, and intermediate-risk myelodysplastic syndromes. Clin Cancer Res 25: 6976-6985, 2019.

11. Kawai T, Takayanagi T,Forrester SJ, Preston KJ, Obama T, Tsuji T, Kobayashi T, Boyer MJ, Cooper HA, Kwok HF, et al: Vascular ADAM17 (a Disintegrin and Metalloproteinase Domain 17) is required for angiotensin II/ $\beta$-aminopropionitrile-induced abdominal aortic aneurysm. Hypertension 70: 959-963, 2017.

12. Kurihara T, Shimizu-Hirota R, Shimoda M, Adachi T, Shimizu H, Weiss SJ, Itoh H, Hori S, Aikawa N and Okada Y: Neutrophil-derived matrix metalloproteinase 9 triggers acute aortic dissection. Circulation 126: 3070-3080, 2012.

13. Nagashima H, Uto K, Sakomura Y, Aoka Y, Sakuta A, Aomi S, HagiwaraN,KawanaMand KasanukiH: An angiotensin-converting enzyme inhibitor, not an angiotensin II type-1 receptor blocker, prevents beta-aminopropionitrile monofumarate-induced aortic dissection in rats. J Vasc Surg 36: 818-823, 2002.

14. Hong J, Hu Z, Wu Q, Tang C, Hu J, Chen R, Li B and Wang Z: The deregulation of STIM1 and store operative calcium entry impaired aortic smooth muscle cells contractility in aortic medial degeneration. Biosci Rep 39: BSR20181504, 2019.

15. Schmittgen TD and Livak KJ: Analyzing real-time PCR data by the comparative C(T) method. Nat Protoc 3: 1101-1108, 2008.

16. Mallem Y, Holopherne D, Reculeau O, Le Coz O, Desfontis JC and Gogny M: Beta-adrenoceptor-mediated vascular relaxation in spontaneously hypertensive rats. Auton Neurosci 118: 61-67, 2005.

17. Shirai K, Song M, Suzuki J, Kurosu T, Oyama T, Nagayama D, Miyashita Y, Yamamura S and Takahashi M: Contradictory effects of $\beta 1$ - and $\alpha 1$-aderenergic receptor blockers on cardio-ankle vascular stiffness index (CAVI)-CAVI independent of blood pressure. J Atheroscler Thromb 18: 49-55, 2011.

18. Thomas P and Dasgupta I: The role of the kidney and the sympathetic nervous system in hypertension. Pediatr Nephrol 30: 549-560, 2015.

19. Parati $G$ and Esler M: The human sympathetic nervous system: Its relevance in hypertension and heart failure. Eur Heart J 33: 1058-1066, 2012.

20. Eichmann A and Brunet I: Arterial innervation in development and disease. Sci Transl Med 6: 252ps259, 2014.

21. Woolgar JR and Scott TM: The relationship between innervation and arterial structure in late prenatal and early postnatal development of the rat jejunal artery. J Anat 167: 57-70, 1989.

22. Hernigou J, Dakhil B, Belmont L, Couffinhal JC and Bagan P: Sleep apnea syndrome and abdominal aortic aneurysm: Study of the prevalence of sleep apnea syndrome in patients with aneurysm and research of association. Clinical study on 52 patients. J Med Vasc 42: 162-169, 2017 (In French).

23. Sampol G, Romero O, Salas A, Tovar JL, Lloberes P, Sagalés T and Evangelista A: Obstructive sleep apnea and thoracic aorta dissection. Am J Respir Crit Care Med 168: 1528-1531, 2003.

24. Yanagi H, Imoto K, Suzuki S, Uchida K, Masuda $M$ and Miyashita A: Acute aortic dissection associated with sleep apnea syndrome. Ann Thorac Cardiovasc Surg 19: 456-460, 2013.

25. Bradley TD and Floras JS: Obstructive sleep apnoea and its cardiovascular consequences. Lancet 373: 82-93, 2009.

26. Schiza SE, Mermigkis C and Bouloukaki I: The effect of obstructive sleep apnea syndrome and snoring severity to intima-media thickening of carotid artery. Sleep Breath 19: 25-27, 2015. 
27. Ruijtenbeek K, le Noble FA, Janssen GM, Kessels CG, Fazzi GE, Blanco CE and De Mey JG: Chronic hypoxia stimulates periarterial sympathetic nerve development in chicken embryo. Circulation 102: 2892-2897, 2000.

28. Angouras DC, Dosios TJ, Dimitriou CA, Chamogeorgakis TP Rokkas CK, Manos TA and Sokolis DP: Surgical thoracic sympathectomy induces structural and biomechanical remodeling of the thoracic aorta in a porcine model. J Surg Res 172 : 68-76, 2012.

29. Brady AR, Thompson SG, Fowkes FG, Greenhalgh RM and Powell JT; UK Small Aneurysm Trial Participants: Abdominal aortic aneurysm expansion: Risk factors and time intervals for surveillance. Circulation 110: 16-21, 2004.

30. Ma J, Zhang L, He G, Tan X, Jin X and Li C: Transcutaneous auricular vagus nerve stimulation regulates expression of growth differentiation factor 11 and activin-like kinase 5 in cerebral ischemia/reperfusion rats. J Neurol Sci 369: 27-35, 2016.

31. Aspalter IM, Gordon E, Dubrac A, Ragab A, Narloch J, Vizán P, Geudens I, Collins RT, Franco CA, Abrahams CL, et al: Alk1 and Alk5 inhibition by Nrp1 controls vascular sprouting downstream of Notch. Nat Commun 6: 7264, 2015.

32. Thomas M, Docx C, Holmes AM, Beach S, Duggan N, England K, Leblanc C, Lebret C, Schindler F, Raza F, et al: Activin-like kinase 5 (ALK5) mediates abnormal proliferation of vascular smooth muscle cells from patients with familial pulmonary arterial hypertension and is involved in the progression of experimental pulmonary arterial hypertension induced by monocrotaline. Am J Pathol 174: 380-389, 2009.
33. Holm TM, Habashi JP, Doyle JJ, Bedja D, Chen Y, van Erp C, Lindsay ME, Kim D, Schoenhoff F, Cohn RD, et al: Noncanonical TGF $\beta$ signaling contributes to aortic aneurysm progression in Marfan syndrome mice. Science 332: 358-361, 2011.

34. Derynck R and Zhang YE: Smad-dependent and Smad-independent pathways in TGF-beta family signalling. Nature 425: 577-584, 2003

35. Schmit BM, Yang P, Fu C, DeSart K, Berceli SA and Jiang Z: Hypertension overrides the protective effect of female hormones on the development of aortic aneurysm secondary to Alk5 deficiency via ERK activation. Am J Physiol Heart Circ Physiol 308: H115-H125, 2015.

36. Carta L, Smaldone S, Zilberberg L, Loch D, Dietz HC, Rifkin DB and Ramirez F: p38 MAPK is an early determinant of promiscuous Smad2/3 signaling in the aortas of fibrillin-1 (Fbn1)-null mice. J Biol Chem 284: 5630-5636, 2009.

37. Fabiani ME, Sourial M, Thomas WG, Johnston CI, Johnston CI and Frauman AG: Angiotensin II enhances noradrenaline release from sympathetic nerves of the rat prostate via a novel angiotensin receptor: Implications for the pathophysiology of benign prostatic hyperplasia. J Endocrinol 171: 97-108, 2001.

38. Li JS, Li HY, Wang L, Zhang L and Jing ZP: Comparison of $\beta$-aminopropionitrile-induced aortic dissection model in rats by different administration and dosage. Vascular 21: 287-292, 2013.

(7) (8) This work is licensed under a Creative Commons Attribution 4.0 International (CC BY-NC 4.0) License 